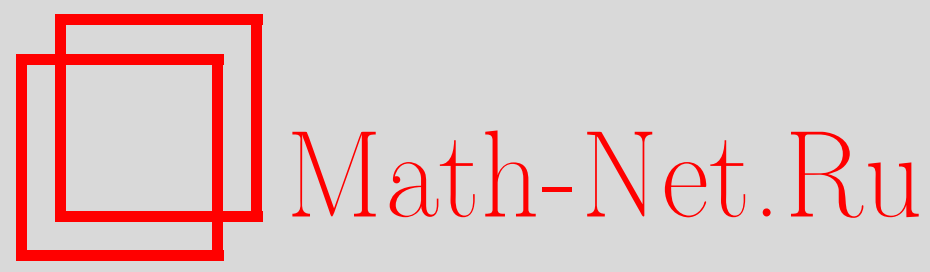

Р. С. Исмагилов, Ультраметрические пространства и связанные с ними гильбертовы пространства, Матем. заметки, 1997, том 62, выпуск 2, 223-237

DOI: https://doi.org/10.4213/mzm1607

Использование Общероссийского математического портала Math-Net.Ru подразумевает, что вы прочитали и согласны с пользовательским соглашением http://www . mathnet.ru/rus/agreement

Параметры загрузки:

IP : 107.22 .136 .117

26 апреля 2023 г., 15:49:22

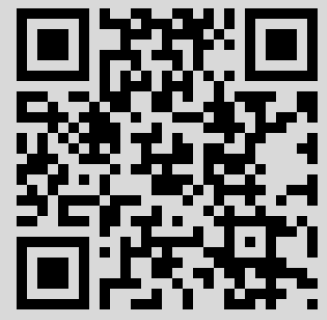




\section{УЛЬТРАМЕТРИЧЕСКИЕ ПРОСТРАНСТВА \\ И СВЯЗАННЫЕ С НИМИ ГИЛЬБЕРТОВЫ ПРОСТРАНСТВА}

\section{Р. С. Исмагилов}

В работе приводятся простые доказательства вложимости ультраметрических пространств в гильбертовы. Основная часть работы посвящена ультраметрическим пространствам, названным вполне-бесконечными. Рассматриваются связанные с ними гильбертовы пространства, автоморфизмы вполне-бесконечных пространств и соответствующие им линейные операторы.

Библиография: 7 названий.

\section{Введение}

Метрическое пространство $(X, d)$ называется ультраметрическим, если

$$
d(x, y) \leqslant \max \{d(x, z), d(z, y)\}, \quad x, y, z \in X .
$$

Известно, что оно допускает изометрическое вложение в вещественное гильбертово пространство. Этому вопросу посвящены работы И.А. Вестфрида и А.Ф. Тимана [1], [2]. Несколько ранее в работах автора [3], [4] было доказано, что для ультраметрических пространств выполняется критерий вложимости в гильбертово пространство; впрочем, само утверждение о вложимости не было сформулировано явно. В $\S 1$ предлагаемой работы мы воспроизведем этот результат, несколько усовершенствовав изложение. Мы приведем также другое доказательство вложимости. Оба доказательства просты и отличаются от доказательств Вестфрида и Тимана.

Затем мы рассматриваем ультраметрические пространства, названные вполне-бесконечньми. Недискретное пространство этого класса не локально компактно, что препятствует построению привычных объектов функционального анализа (меры, инвариантной относительно изометрических преобразований, функциональных пространств и т.д.). Тем не менее, мы построим некоторую алгебру функций на вполне-бесконечном пространстве $(X, d)$ и введем в ней семейство скалярных произведений, инвариантных относительно любой изометрии. Получается набор гильбертовых пространств - аналог пространств типа $L_{2}$ на пространствах с мерой.

В $\S 3$ вводятся автоморфизмы вполне-бесконечных пространств: аналоги преобразования пространств с мерой, оставляющего меру квазиинвариантной. Определяется якобиан автоморфизма - аналог производной Радона-Никодима преобразованной меры

Работа выполнена при финансовой поддержке Американского математического общества. 
относительно исходной. Автоморфизму ставится в соответствие линейньй оператор в упомянутой алгебре функций, он задается заменой переменной с последующим умножением на некоторую степень якобиана (стандартный прием в теории преобразований пространств с мерой). Для изометрических преобразований якобиан равен 1 , а соответствующий оператор унитарен в каждом из указанных гильбертовых пространств. Отметим, что некоторые автоморфизмы с якобианом, отличным от тождественной единищы, порож дают унитарные операторы в некоторых из упомянутых гильбертовых пространств. Мы построим группу автоморфизмов с этим свойством.

В качестве примера рассматривается поле $\mathbb{F}$ формальных степенных рядов с коэффициентами из бесконечного поля и проективная прямая $\mathbb{P}^{1}(\mathbb{F})$ с естественной метрикой $d$. Естественное действие группы $\mathrm{GL}_{2}(\mathbb{F})$ на $\mathbb{P}^{1}(\mathbb{F})$ задает автоморфизмы вполне-бесконечного пространства $\left(\mathbb{P}^{1}(\mathbb{F}), d\right)$. Это приводит к унитарным представлениям группы $\mathrm{GL}_{2}(\mathbb{F})$ в указанных гильбертовых пространствах (представления группы $\mathrm{SL}_{2}(\mathbb{F})$ рассмотрены в [3]-[6]). Эти групшы не локально компактны. Подчеркнем, что в этой, сугубо элементарной, работе не используются какие-либо сведения о представлениях групп (термин “представление” поясняется в п. 3.1).

\section{§1. Изометрические вложения ультраметрических пространств}

Мы покажем, что ультраметрическое пространство изометрически вкладывается в пространство функций. Затем приведем два простых доказательства вложимости в гильбертово пространство. Как упоминалось во введении, другие изложения этих вопросов содержатся в работах [1]-[5], [7].

Если $(X, d)$ - произвольное метрическое пространство, то шар радиуса $r \geqslant 0$ с центром $a \in X$ определяется равенством

$$
U_{a}^{r}=\{x \mid x \in X, d(x, a) \leqslant r\} .
$$

Для ультраметрического пространства $(X, d)$ шары $U_{a}^{r}, U_{b}^{r}$ совпадают при $d(a, b) \leqslant r$ и не пересекаются при $d(a, b)>r$, это следует из (1). Положим

$$
\operatorname{diam} X=\sup \{d(x, y) \mid x, y \in X\} .
$$

1. Универсальные метрические пространства. Начнем с примеров ультраметрических пространств, состоящих из функций.

1) Возьмем произвольное множество $A$, содержащее более одного элемента, и ограниченное числовое множество $E \subset(0, \infty)$. Через $A^{E}$ обозначим множество всех отображений $\xi: E \rightarrow A$. Введем в $A^{E}$ метрику $\delta$ по формулам $\delta\left(\xi_{1}, \xi_{2}\right)=\sup \left\{t \mid \xi_{1}(t) \neq \xi_{2}(t)\right\}$ при $\xi_{1} \neq \xi_{2}, \delta\left(\xi_{1}, \xi_{1}\right)=0$. Получим ультраметрическое пространство $\left(A^{E}, \delta\right)$.

2) Возьмем такое же множество $A$ и неограниченное сверху множество $E \subset(0, \infty)$. Зафиксируем отображение $\eta: E \rightarrow A$. Обозначим через $A_{\eta}^{E}$ множество всех отображений $\xi: E \rightarrow A$ таких, что $\xi(t)=\eta(t)$ для достаточно больших $t \in E$. Введем метрику $\delta$ так же, как и вьше. Получим ультраметрическое пространство $\left(A_{\eta}^{E}, \delta\right)$.

Следующие предложения выявляют свойство “универсальности” построенных пространств.

ПРЕДЛОЖЕНИЕ 1. Любое ультраметрическое пространство $(X, d)$, диаметр которого конечен, изометрически вкладывается в некоторое $\left(A^{E}, \delta\right)$. Если пространство $X$ конечно, то множества $A, E$ также можсно выбрать конечными. 
ДокАЗАТЕЛЬСТво. Пусть $E$ - множество всех положительных значений, принимаемых метрикой $d$. Через $A$ обозначим множество всех шаров пространства $X$. Рассмотрим пространство $\left(A^{E}, \delta\right)$. Каждой точке $x \in X$ поставим в соответствие функцию $\xi_{x} \in A^{E}$ вида $\xi_{x}(t)=U_{x}^{t}, t \in E$. Покажем, что полученное отображение $x \mapsto \xi_{x}, x \in X$, изометрично. Для этого возьмем точки $x, y \in X, x \neq y$. Пусть $r=d(x, y)$. Если $t \in E$, $t<r$, то $U_{x}^{t} \cap U_{y}^{t}=\varnothing ;$ если $t \geqslant r$, то $U_{x}^{t}=U_{y}^{t}$. Итак,

$$
\delta\left(\xi_{x}, \xi_{y}\right)=\sup \left\{t \mid \xi_{x}(t) \neq \xi_{y}(t)\right\}=r=d(x, y),
$$

что и требовалось.

ПРЕДЛОЖЕНИЕ 2. Любое ультраметрическое пространство бесконечного диаметра изометрически вкладьвается в некоторое $\left(A_{\eta}^{E}, \delta\right)$.

Доказывается аналогично.

В работе [5, лемма 9] показано, что любое конечное ультраметрическое пространство изометрически вкладьвается в поле формальных степенных рядов. Другая конструкция предложена в работе Вестфрида [2]. Изометрические вложения в другие пространства, необязательно ультраметрические, рассмотрены в [7].

2. Вложение в гильбертово пространство. Известно и легко доказывается, что метрическое пространство допускает изометрическое вложение в вещественное гильбертово пространство, если этим свойством обладает любое его конечное подпространство. Поэтому мы ограничимся конечными пространствами.

Итак, пусть $(X, d)$ - конечное ультраметрическое пространство, $c_{0}>c_{1}>\cdots>c_{m}=0$ множество всех значений метрики $d$. Наша цель - изометрически вложить $(X, d)$ в вещественное гильбертово пространство.

ПЕРВОЕ ДОКАЗАТЕЛЬСТВО ВЛОЖИМОСТИ. Не ограничивая общности, будем считать, что для любой точки $x \in X$ шары $U_{x}^{c_{k}}, 1 \leqslant k \leqslant m$, попарно различны, ибо согласно предложению 1 любое $X$ вкладывается в пространство с этим свойством. Обозначим через $H(X)$ вещественное гильбертово пространство, ортонормированным базисом которого служит набор всех шаров пространства $(X, d)$, отличных от $X$. Каждый такой шар имеет вид $U_{a}^{c_{k}}, a \in X, 1 \leqslant k \leqslant m$. Определим отображение $\varphi: X \rightarrow H(X)$ формулой

$$
x \mapsto \varphi(x)=\sum_{k=1}^{m} \lambda_{k} U_{x}^{c_{k}}, \quad \lambda_{k}=\sqrt{\frac{c_{k-1}^{2}-c_{k}^{2}}{2}} .
$$

ПРЕДЛоЖенИЕ 3. Отображение $\varphi: X \rightarrow H(X)$, заданное формулой (2), изометрично.

ДокАЗАТЕЛЬСТво. Возьмем точки $x, y \in X, x \neq y$. Тогда $d(x, y)=c_{p}$, где $0 \leqslant p<m$, и

$$
\varphi(x)=\sum_{k=1}^{m} \lambda_{k} U_{x}^{c_{k}}, \quad \varphi(y)=\sum_{j=1}^{m} \lambda_{j} U_{y}^{c_{j}} .
$$


Шары $U_{x}^{c_{k}}, U_{y}^{c_{j}}$ совпадают тогда и только тогда, когда $k=j, d(x, y) \leqslant c_{k}$; последнее неравенство равносильно неравенству $k \leqslant p$. Следовательно, $\left(U_{x}^{c_{k}}, U_{y}^{c_{k}}\right)=1$ при $k \leqslant p$, $\left(U_{x}^{c_{k}}, U_{y}^{c_{j}}\right)=0$ при $k \neq j$ и $p<k=j$. Поэтому

$$
\begin{aligned}
|\varphi(x)-\varphi(y)|^{2} & =|\varphi(x)|^{2}+|\varphi(y)|^{2}-2(\varphi(x), \varphi(y))=2 \sum_{k=1}^{m} \lambda_{k}^{2}-2 \sum_{k=1}^{p} \lambda_{k}^{2} \\
& =2 \sum_{k=p+1}^{m} \lambda_{k}^{2}=\sum_{k=p+1}^{m}\left(c_{k-1}^{2}-c_{k}^{2}\right)=c_{p}^{2}=d^{2}(x, y) .
\end{aligned}
$$

Итак, $|\varphi(x)-\varphi(y)|=d(x, y)$. Этим доказано предложение 3 .

ВТОРОЕ ДОКАЗАТЕЛЬСТВо ВЛОЖИмоСТИ. Доказательство, которое будет сейчас приведено, содержится, по-сушеству, в [3, лемма 10], а также в [4, лемма 2]. Мы лишь упростим изложение.

Пусть $(X, d)$ - произвольное метрическое пространство, $L$ - линейное пространство всех функций $f: X \rightarrow \mathbb{R}$, имеющих конечньй носитель. Определим в $L$ билинейную форму

$$
\left[f_{1}, f_{2}\right]=-\sum_{x, y \in X} d^{2}(x, y) f_{1}(x) f_{2}(y) .
$$

ПРЕДЛОЖЕНИЕ 4. Метрическое пространство $(X, d)$ допускает изометрическое вложсение в гильбертово пространство в том и только том случае, если квадратичная форма $[f, f]$ неотрицательно определена на подпространстве

$$
L_{0}=\left\{f \mid f \in L, \sum_{x \in X} f(x)=0\right\}
$$

m.e. $[f, f] \geqslant 0$ npu $f \in L_{0}$.

ДокАЗАТЕЛЬСТво. Пусть, как и раньше, $X$ конечно, $n$ - число его элементов. Пусть $[f, f] \geqslant 0$ при $f \in L_{0}$. Положим $L_{00}=\left\{f \mid f \in L_{0},[f, f]=0\right\}$. Тогда $L_{00}-$ линейное подпространство в $L_{0}$ и в факторпространстве $H=L_{0} / L_{00}$ возникает положительно определенное скалярное произведение $\frac{1}{2}\left[f_{1}, f_{2}\right]$. Образ элемента $f \in L_{0}$ при естественном отображении $L_{0} \rightarrow H$ обозначим через $\bar{f}$. Для любой точки $a \in X$ введем функцию $\delta_{a}$ вида $\delta_{a}(x)=0$ при $x \neq a, \delta_{a}(a)=1$. Возьмем также функцию $f^{0}(x)=1, x \in X$. Заметим, что $\delta_{a}-\frac{1}{n} f^{0} \in L_{0}$, тем самым, определено отображение $\psi: X \rightarrow H$ формулой $\psi(a)=\overline{\delta_{a}-\frac{1}{n} f^{0}}$. Для любых точек $x, y \in X$ имеем $|\psi(x)-\psi(y)|^{2}=\frac{1}{2}\left[\delta_{x}-\delta_{y}, \delta_{x}-\delta_{y}\right]=d^{2}(x, y)$. Итак, отображение $\psi: X \rightarrow H$ изометрично.

Мы доказали, что условие $[f, f] \geqslant 0, f \in L_{0}$, достаточно для вложимости пространства $(X, d)$ в гильбертово пространство. Необходимость этого условия очевидна. Предложение 4 доказано.

Обратимся к ультраметрическому пространству $(X, d)$. Пусть оно конечно и $c_{0}>\cdots>c_{m}=0-$ множество всевозможных значений, принимаемых метрикой $d$. Для любого $k=0,1, \ldots, m$ пространство $X$ является объединением $l_{k}$ попарно не 
пересекающихся шаров радиуса $c_{k}$. Обозначим эти шары через $U(k, i), 1 \leqslant i \leqslant l_{k}$. Заметим, что $l_{0}=1, l_{m}=|X|$. Введем квадратичные формы

$$
S_{k}(f)=\sum_{i=1}^{l_{k}}\left|\sum_{x \in U(k, i)} f(x)\right|^{2}, \quad f \in L, \quad 0 \leqslant k \leqslant m .
$$

В частности,

$$
S_{0}(f)=\left|\sum_{x \in X} f(x)\right|^{2}, \quad S_{m}(f)=\left|\sum_{x \in X} f(x)\right|^{2} .
$$

ПРЕДЛОЖЕНИЕ 5. Справедливо равенство

$$
-\sum_{x, y \in X} d^{2}(x, y) f(x) f(y)=-c_{0}^{2} S_{0}(f)+\sum_{k=1}^{m}\left(c_{k-1}^{2}-c_{k}^{2}\right) S_{k}(f), \quad f \in L .
$$

ДоКАЗАТЕЛЬСТВо. Предварительно для любого $k=0,1, \ldots, m$ подсчитаем сумму

$$
\sum_{d(x, y) \leqslant c_{k}} f(x) f(y) .
$$

Неравенство $d(x, y) \leqslant c_{k}$ равносильно тому, что точки $x, y$ лежат в одном из шаров $U(k, i)$. Отсюда

$$
\sum_{d(x, y) \leqslant c_{k}} f(x) f(y)=\sum_{i=1}^{l_{k}} \sum_{x, y \in U(k, i)} f(x) f(y)=\sum_{i=1}^{l_{k}}\left|\sum_{x \in U(k, i)} f(x)\right|^{2}=S_{k}(f) .
$$

Следовательно,

$$
\begin{aligned}
-\sum_{x, y \in X} d^{2}(x, y) f(x) f(y) & =-\sum_{k=0}^{m-1} c_{k}^{2} \sum_{d(x, y)=c_{k}} f(x) f(y) \\
& =\sum_{k=0}^{m-1} c_{k}^{2}\left(\sum_{d(x, y) \leqslant c_{k}} f(x) f(y)-\sum_{d(x, y) \leqslant c_{k+1}} f(x) f(y)\right) \\
& =-\sum_{k=0}^{m-1} c_{k}^{2}\left(S_{k}(f)-S_{k+1}(f)\right) \\
& =-c_{0}^{2} S_{0}(f)+\sum_{k=1}^{m}\left(c_{k-1}^{2}-c_{k}^{2}\right) S_{k}(f) .
\end{aligned}
$$

Предложение 5 доказано.

Из (3) следует, что

$$
-\sum_{x, y \in X} d^{2}(x, y) f(x) f(y) \geqslant c_{m-1}^{2} \sum_{x \in X}|f(x)|^{2}, \quad f \in L_{0} .
$$

Отсюда получаем, используя предложение 4 , что $(X, d)$ вложимо в гильбертово пространство. 


\section{§ 2. Вполне-бесконечные пространства}

Пусть $(X, d)$ - ультраметрическое пространство, $E=d(X \times X)$ - множество значений метрики $d$. Назовем пространство $(X, d)$ вполне-бесконечным, если для любого положительного $r \in E$ и любого конечного набора точек $\left\{x_{i}, 1 \leqslant i \leqslant n\right\} \subset X$ такого, что $d\left(x_{i}, x_{j}\right)=r$ при $i \neq j$, сушествует такая точка $x_{n+1} \in X$, что $d\left(x_{n+1}, x_{i}\right)=r$, $1 \leqslant i \leqslant n$. Тем самым, множество $\left\{x_{i}, 1 \leqslant i \leqslant n\right\}$ может быть включено в бесконечное множество равноотстоящих точек. Если при этом множество $E$ дискретно, т.е. состоит из изолированных точек, то назовем $(X, d)$ пространством дискретного типа; если $E$ конечно, то отнесем $(X, d)$ к конечному типу. Если, наконец, $E$ является плотньг подмножеством отрезка $[0, a], 0<a \leqslant \infty$, то назовем $(X, d)$ пространством непрерывного типа.

В п. 2.1 мы рассмотрим некоторые простые свойства вполне-бесконечных пространств, а в п. 2.2 введем алгебры функций и гильбертовы пространства функций на них.

1. Свойства вполне-бесконечных пространств. В следующих леммах $(X, d)$ является вполне-бесконечньп пространством, а $E$ - множеством значений метрики $d$.

Лемма 1. Пусть $(Y, \delta)$ - конечное ультраметрическое пространство, $F$ - множество значений метрики $\delta$, причем $F \subset E$. Пусть $Y_{0} \subset Y$. Тогда любое изометрическое отображсене $Y_{0} \rightarrow X$ можно продолжить до изометрического отображения $Y \rightarrow X$.

ДокАЗАТЕЛЬСТво. Пусть $m$ - число точек множества $F$. Применим индукцию по $m$. Если $m=2$, то утверждение леммы вытекает из определения вполне-бесконечного пространства. Пусть теперь $m>2$ и множество $F$ состоит из чисел $r_{1}>\cdots>r_{m}=0$. Шары радиуса $r_{2}$ образуют разбиение $Y=Y_{1} \cup \cdots \cup Y_{p}, p>1$. По индуктивному допущению утверждение леммы верно для любого шара $Y_{i}$. Отсюда легко вьводится, что оно верно и для $Y$.

Лемма 2. Для любого конечного множества $S \subset X$ существует такое множество $S^{0}$, что $S \subset S^{0} \subset X u\left(S^{0}, d\right)$ - вполне-бесконечное пространство конечного muna.

Доказательство, как и выше, основано на индукции по числу точек в множестве значений метрики $d$ на подпространстве $S$, при этом используется лемма 1 . Несложные подробности опускаем.

2. Алгебры функций и гильбертовы пространства функций. Начнем с простого замечания. Пусть $(X, d)$ - произвольное метрическое пространство. Для любой функции $\varphi:[0, \infty)^{n} \rightarrow \mathbb{C}, n=1,2, \ldots$, и любого набора точек $a_{i} \in X, 1 \leqslant i \leqslant n$, возникает функция $\varphi\left(d\left(x, a_{1}\right), \ldots, d\left(x, a_{n}\right)\right), x \in X$. Взяв набор функций $\varphi$, можно образовать алгебру функций на $X$, порожденную функциями указанного вида. В следующем предложении будет показано, что для ультраметрического пространства эта конструкция имеет интересную особенность.

ПРЕДЛОЖЕНИЕ 6. Пусть $(X, d)$ - ультраметрическое пространство, $a_{i} \in X$, $1 \leqslant i \leqslant n, \varphi\left(t_{1}, \ldots, t_{n}\right), t_{i} \geqslant 0,-$ комплекснозначная функция. Тогда существуют 
функиии $\psi_{i}(t), t \geqslant 0,1 \leqslant i \leqslant n$, обладающие следующими свойствами:

1) справедливо равенство

$$
\varphi\left(d\left(x, a_{1}\right), \ldots, d\left(x, a_{n}\right)\right)=\sum_{i=1}^{n} \psi_{i}\left(d\left(x, a_{i}\right)\right), \quad x \in X ;
$$

2) существуют такие точки $0=t_{0}<\cdots<t_{m}=1$, что каждая из функиий $\psi_{i}(t), t \in\left[t_{i-1}, t_{i}\right]$, представляется в виде линейной комбинации функций, полученных из функиии $\varphi\left(t_{1}, \ldots, t_{n}\right)$ заменой $t_{i}$ на $t$ для некоторых значений $i$ и заменой $t_{i}$ на константы $t_{i}^{0}$ для остальных значений $i$.

ДокАЗАТЕльСтво. Пусть сначала $n=2$, т.е. дана функция $\varphi\left(d\left(x, a_{1}\right), d\left(x, a_{2}\right)\right)$, $x \in X$. Положим $r=d\left(a_{1}, a_{2}\right)$. Легко видеть, что точка $\left(d\left(x, a_{1}\right), d\left(x, a_{2}\right)\right)$ лежит в множестве $F \subset \mathbb{R}^{2}$, которое является объединением отрезков $\left\{\left(t_{1}, r\right), 0 \leqslant t_{1} \leqslant r\right\}$, $\left\{\left(r, t_{2}\right), 0 \leqslant t_{2} \leqslant r\right\},\{(t, t), r \leqslant t\}$. Осталось подобрать такие функции $\psi_{i}\left(t_{i}\right), i=1,2$, что $\varphi\left(t_{1}, t_{2}\right)=\psi_{1}\left(t_{1}\right)+\psi_{2}\left(t_{2}\right)$ при $\left(t_{1}, t_{2}\right) \in F$. Достаточно положить $\psi_{1}\left(t_{1}\right)=\varphi\left(t_{1}, r\right)$, $0 \leqslant t_{1} \leqslant r ; \psi_{1}\left(t_{1}\right)=\varphi\left(t_{1}, t_{1}\right), t_{1} \geqslant r ; \psi_{2}\left(t_{2}\right)=\varphi\left(r, t_{2}\right)-\varphi(r, r), 0 \leqslant t \leqslant r ; \psi_{2}\left(t_{2}\right)=0$, $t_{2} \geqslant r$. Этим доказано наше предложение при $n=2$. Далее применяем индукцию по $n$. При $n \geqslant 3$, используя индуктивное предположение, мы можем представить функцию $\varphi\left(d\left(x, a_{1}\right), \ldots, d\left(x, a_{n-1}\right), t_{n}\right), x \in X, t_{n} \geqslant 0$, в виде суммы функций $h_{i}\left(d\left(x, a_{i}\right), t_{n}\right)$, $1 \leqslant i \leqslant n-1$. Каждую из функций $h_{i}\left(d\left(x, a_{i}\right), d\left(x, a_{n}\right)\right)$ можно представить в виде суммы функций $g_{i j}\left(d\left(x, a_{k}\right)\right)$. Это приводит к равенству $(4)$. Предложение 6 доказано.

Пусть теперь $(X, d)$ - вполне-бесконечное пространство дискретного или непрерывного типа. Мы введем алгебру функций на $X$, следуя образцу, описанному в начале этого пункта. Для простоты ограничимся случаем $\operatorname{diam} X=1$.

Пусть сначала $(X, d)$ - пространство дискретного типа. Множество значений метрики $d$ состоит либо из чисел $1=c_{0}>\cdots>c_{m}=0$, либо из чисел $1=c_{0}>c_{1}>\cdots$, $c_{k} \rightarrow 0$ при $k \rightarrow \infty$. Обозначим через $f_{a}^{k}$ индикатор шара $U_{a}^{c_{k}}, a \in X$. Заметим, что равенство $f_{a}^{k}=f_{b}^{m}$ равносильно условиям $k=m, d(a, b) \leqslant c_{k}$. Через $B(X)$ обозначим множество функций $f(x), x \in X$, представимых в виде конечных линейных комбинаций функций $f_{a}^{k}$. Ясно, что $B(X)$ - алгебра относительно умножения функций. Важно отметить, что набор $\left\{f_{a}^{k}\right\}$ линейно независим, ибо любой шар $U_{a}^{c_{k}}$ является объединением бесконечного набора шаров радиуса $c_{k+1}$. Добавим, что $B(X)$ есть линейная оболочка функций вида $\varphi(d(x, a))$, где функция $\varphi(t), 0 \leqslant t \leqslant 1$, постоянна в окрестности нуля.

Зафиксируем числа $\beta_{k}, k=0,1, \ldots$, и введем в $B(X)$ скалярное произведение $(\cdot, \cdot)$, задав его на базисе $\left\{f_{a}^{k}\right\}$ равенствами

$$
\left(f_{a}^{k}, f_{b}^{m}\right)= \begin{cases}0, & f_{a}^{k} \neq f_{b}^{m}, \\ \beta_{k}, & f_{a}^{k}=f_{b}^{m} .\end{cases}
$$

Гильбертово пространство, полученное из $B(X)$ пополнением по норме $\sqrt{(f, f)}$, обозначим через $H(X)\left(H^{\left\{\beta_{k}\right\}}(X)\right.$ либо $\left.H^{\left\{\beta_{k}\right\}}\right)$. Итак, для пространства $(X, d)$ дискретного типа гильбертово пространство построено.

Пусть теперь $(X, d)$ - пространство непрерьвного типа, причем $\operatorname{diam} X=1$. Тем самым, множество значений метрики $d$ плотно в отрезке $[0,1]$. Построим алгебру функций и гильбертовы пространства, связанные с $(X, d)$. 
Назовем функцию $\varphi:[0,1] \rightarrow \mathbb{C}$ кусочно-лладкой, если она непрерывна, имеет непрерьвную производную в $[0,1]$, за возможным исключением конечного набора точек, причем производная ограничена на области ее определения. Обозначим через $B(X)$ множество всех функций вида

$$
f(x)=\sum_{k=1}^{N} \varphi_{k}\left(d\left(x, a_{k}\right)\right), \quad x \in X
$$

где $a_{k} \in X, 1 \leqslant k \leqslant N, \varphi_{k}(t), 0 \leqslant t \leqslant 1,-$ кусочно-гладкие функции. Из предложения 6 следует, что $B(X)$ - алгебра функций.

Введем в $B(X)$ скалярное произведение. Грубо говоря, мы определим его путем предельного перехода от подпространств $Y \subset X$, принадлежащих дискретному типу. Сейчас будет дано подробное описание.

Зафиксируем число $\beta \geqslant 0$ и непрерьвную неотрицательную функцию $\theta(t), 0 \leqslant t \leqslant 1$, последняя порождает меру $\theta(t) d t$ на отрезке $[0,1]$. Возьмем любое подпространство конечного типа $Y \subset X$, пусть $c_{0}>\cdots>c_{m}=0$ - множество значений метрики $d$ на $Y$. Возьмем числа $\beta_{k}, 0 \leqslant k \leqslant m$, и введем в алгебре $B(Y)$ скалярное произведение, как описано выше (см. (5)). Это скалярное произведение обозначим через $(\cdot, \cdot)_{Y}^{\left\{\beta_{k}\right\}}$. Будем варьировать пространство $Y$ и числа $\left\{\beta_{k}\right\}$ так, чтобы число $\beta_{0}$ стремилось к $\beta$, мера $\tau_{Y}^{\left\{\beta_{k}\right\}}$, предписьваюшая каждой точке $c_{k}$ вес $\beta_{k}\left(c_{k}-c_{k-1}\right)^{2}$, стремилась к мере $\theta(t) d t$ в слабой топологии и, наконец, величина $\max _{k}\left(c_{k}-c_{k+1}\right)$ стремилась к 0 .

Теперь мы готовы осуществить обешанный предельньй переход, что позволит построить скалярное произведение в $B(X)$. Возьмем любые две функции $f, h \in B(X)$. Таким образом,

$$
f(x)=\sum_{i} \varphi_{i}\left(d\left(x, a_{i}\right)\right), \quad h(x)=\sum_{i} \psi_{i}\left(d\left(x, b_{i}\right)\right), \quad x \in X,
$$

где $a_{i}, b_{i} \in X, \varphi_{i}, \psi_{i}-$ кусочно-гладкие функции, обе суммы конечны. Возьмем подпространство $Y \subset X$ конечного типа, содержащее все точки $a_{i}, b_{i}$ (оно сушествует в силу леммы 2 ). Возьмем также числа $\beta_{k}>0$ и введем в $B(Y)$ скалярное произведение $(\cdot, \cdot)_{Y}^{\left\{\beta_{k}\right\}}$, как указано вьше. Ясно, что функции $f|Y, h| Y$ принадлежат пространству $B(Y)$, а потому определено их скалярноепроизведение. Будем варьировать $Y$ и $\left\{\beta_{k}\right\}$, как объяснялось вьше. Положим

$$
(f, h) \stackrel{\text { def }}{=} \lim (f|Y, h| Y)_{Y}^{\left\{\beta_{k}\right\}} .
$$

(Строгое изложение приведенной конструкции потребовало бы языка фильтров и т.п., мы предпочли простое описание.)

ЛЕмма 3. Предел (6) существует для любых функиий из В(X). В частности, если

$$
f(x)=\varphi(d(x, a)), \quad h(x)=\psi(d(x, b)), \quad x \in X
$$

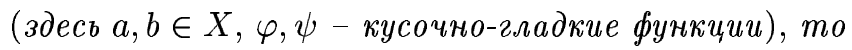

$$
(f, h)=\beta \varphi(1) \overline{\psi(1)}+\int_{r}^{1} \varphi^{\prime}(t) \overline{\psi^{\prime}(t)} \theta(t) d t, \quad r=d(a, b) .
$$


ДокаЗАТЕЛЬСтво. Пусть $f_{x}^{k}$-индикатор шара $\left\{y \mid y \in Y, d(y, x) \leqslant c_{k}\right\}$. Ясно, что

$$
\begin{aligned}
f(x) & =\sum_{k=0}^{m-1} \varphi\left(c_{k}\right)\left(f_{a}^{k}(x)-f_{a}^{k+1}(x)\right)+\varphi\left(c_{m}\right) f_{a}^{m}(x) \\
& =\sum_{k=1}^{m}\left(\varphi\left(c_{k}\right)-\varphi\left(c_{k-1}\right)\right) f_{a}^{k}(x)+\varphi\left(c_{0}\right) f_{a}^{0}, \quad x \in Y .
\end{aligned}
$$

Аналогично записывается функция $h(x)$. Положим $d(a, b)=c_{p}$. Напомним, что $f_{a}^{k}=f_{b}^{i}$ при $k=i, p \leqslant k,\left(f_{a}^{k}, f_{b}^{i}\right)=0$ при $i \neq k$ и при $i=k<p$. Поэтому

$$
(f|Y, h| Y)_{Y}^{\left\{\beta_{k}\right\}}=\sum_{k=p}^{m}\left(\varphi\left(c_{k}\right)-\varphi\left(c_{k-1}\right)\right) \overline{\left(\psi\left(c_{k}\right)-\psi\left(c_{k-1}\right)\right)} \beta_{k}+\varphi\left(c_{0}\right) \overline{\psi\left(c_{0}\right)} \beta_{0} .
$$

Перейдем к пределу, варьируя $Y$ и $\left\{\beta_{k}\right\}$, как объяснялось вьше. Несложное вычисление, использующее равенство Лагранжа $\varphi\left(t_{1}\right)-\varphi\left(t_{2}\right)=\varphi^{\prime}(s)\left(t_{1}-t_{2}\right)$ и аналогичное равенство для $\psi$, приводит к формуле (7). Лемма доказана.

Итак, скалярное произведение в $B(X)$ построено. Пополнение пространства $B(X)$ приводит к гильбертову пространству, обозначим его $H(X)$ (либо $\left.H_{\theta}^{\beta}(X)\right)$. Оно задается числом $\beta \geqslant 0$ и мерой $\theta(t) d t$.

Используя лемму 3 , представим $H_{\theta}^{\beta}(X)$ в виде прямого интеграла гильбертовых пространств. С этой целью для любого $r>0$ обозначим через $H_{r}$ гильбертово пространство, ортонормированным базисом которого служит множество всех шаров радиуса $r$ пространства $X$; в частности, $H_{1}$ - одномерное пространство с базисом $X$. Каждой точке $a \in X$ и каждой кусочно-гладкой функции $\varphi(t), t \in[0,1]$, поставим в соответствие вектор-функцию $r \mapsto h_{a}^{\varphi}(r) \in H_{r}, 0 \leqslant r \leqslant 1$, задав ее равенствами $h_{a}^{\varphi}(r)=\varphi^{\prime}(r) U_{a}^{r}$ при $r<1, h_{a}^{\varphi}(1)=\varphi(1) X$. Введем на $[0,1]$ меру $\sigma$, полагая $d \sigma=\theta(t) d t$ на $[0,1), \sigma\{1\}=\beta$. Семейство $\left\{H_{r}\right\}$, мера $\sigma$ и набор вектор-функций $\left\{h_{a}^{\varphi}\right\}$ определяет прямой интеграл $\mathscr{H}=\int_{0}^{1} H_{r} d \sigma(r)$. Лемма 3 устанавливает естественный изоморфизм гильбертовых пространств $\mathscr{H}$ и $H_{\theta}^{\beta}(X)$.

\section{§3. Автоморфизмы вполне-бесконечных пространств и порожденные ими линейные операторы}

Напомним, что если $(X, \tau)$ - пространство с мерой, то мера называется квазиинвариантной относительно биекиии $x \mapsto x g, x \in X$, если она переходит в эквивалентную меру. В этом случае определена производная $\dot{g}(x)=d \tau(x g) / d \tau(x)$, называемая якобианом преобразования $g$. Биекции с этим свойством образуют группу $\operatorname{Aut}(X, \tau)$. Для любого $\lambda \in \mathbb{C}$ операторы

$$
\left(T^{\lambda}(g) f\right)(x)=(\dot{g}(x))^{\lambda} f(x g), \quad x \in X, \quad g \in \operatorname{Aut}(X, \tau),
$$

образуют линейное представление этой группы в пространстве измеримых функций. При $\operatorname{Re} \lambda=1 / 2$ представление унитарно в пространстве $L_{2}(X, \tau)$.

Цель этого параграфа - построение некоторого аналога, описанного сейчас, для вполне-бесконечных пространств. В этом случае мера на $X$ отсутствует, и в роли $L_{2}(X, \tau)$ выступят построенные выше гильбертовы пространства $H(X)$. 
1. Группа $\operatorname{Aut}(X, d)$. Предварительно напомним, что отображение метрических пространств $f:(X, d) \rightarrow(Y, \delta)$ называется гомотетией, если

$$
\delta\left(f\left(x_{1}\right), f\left(x_{2}\right)\right)=k d\left(x_{1}, x_{2}\right), \quad x_{1}, x_{2} \in X
$$

для некоторого $k>0$. Число $k$ назьвается коэффиииентом гомотетии.

Пусть теперь $(X, d)$ - вполне-бесконечное пространство, принадлежащее дискретному либо непрерывному типу. Предполагается, что $\operatorname{diam} X=1$. Автоморфизмом пространства $(X, d)$ назовем биекцию $x \mapsto x g, x \in X$, удовлетворяющую следующим условиям:

1) для любой функции $f \in B(X)$ функция $x \mapsto f(x g), x \in X$, также принадлежит пространству $B(X)$;

2) для любой точки $x \in X$ существует такая ее окрестность $U_{x}^{r}, r>0$, что отображение $g \mid U_{x}^{r}$ есть гомотетия, тем самым, определена функция $\dot{g}$ на $X$ такая, что $\dot{g}(x)$ есть коэффициент гомотетии отображения $g$ в окрестности точки $x$;

3) введенная функция $\dot{g}(x), x \in X$, принадлежит алгебре $B(X)$.

Функцию $\dot{g}$ назовем якобианом автоморфизма $g$. Из предложения 6 легко вьвести, что для любого $\lambda \in \mathbb{C}$ функция $(\dot{g}(x))^{\lambda}, x \in X$, также принадлежит алгебре $B(X)$.

Группу всех автоморфизмов пространства $(X, d)$ обозначим через $\operatorname{Aut}(X, d)$ (мы опускаем несложную проверку того, что автоморфизмы, действительно, образуют групу). Выполняется равенство $\left(g_{1} g_{2}\right)^{*}(x)=\dot{g}_{1}(x) \dot{g}_{2}\left(x g_{1}\right), x \in X, g_{i} \in \operatorname{Aut}(X, d)$. Из него следует, что для любого $\lambda \in \mathbb{C}$ операторы

$$
\left(T^{\lambda}(g) f\right)(x)=(\dot{g}(x))^{\lambda} f(x g), \quad f \in B(X), \quad g \in \operatorname{Aut}(X, d),
$$

образуют линейное представление группы $\operatorname{Aut}(X, d)$, т.е. $T^{\lambda}(e)=I(e-$ тождественный автоморфизм $), T^{\lambda}\left(g_{1} g_{2}\right)=T^{\lambda}\left(g_{1}\right) T^{\lambda}\left(g_{2}\right), g_{i} \in \operatorname{Aut}(X, d)$.

2. Автоморфизмы, порождающие унитарные операторы. Обозначим через $\operatorname{Iso}(X, d)$ группу всех изометрических биекций пространства $(X, d) ;$ ясно, что $\operatorname{Iso}(X, d) \subset$ $\operatorname{Aut}(X, d)$. Если $g \in \operatorname{Iso}(X, d)$, то $\dot{g}(x)=1, x \in X$, и оператор (8) сохраняет любое из скалярных произведений в $B(X)$, введенных в $\oint 2$. Тем самым, возникает унитарное представление групшы Iso $(X, d)$ в соответствующем гильбертовом пространстве $H(X)$. Цель этого пункта состоит в следующем: для некоторых вполне-бесконечных пространств $(X, d)$ мы построим подгруппу $G(X, d) \subset \operatorname{Aut}(X, d)$, содержащую подгруппу Iso $(X, d)$, но не совпадающую с ней, такую, что при $\lambda>0$ операторы $T^{\lambda}(g)$ будут унитарными в одном из гильбертовых пространств $H(X)$ для всех $g \in G(X, d)(H(X)$ будет зависеть от $\lambda)$. Заметим, что существование автоморфизмов пространства $(X, d)$, не являющихся изометриями и, тем не менее, порождающих унитарные операторы в некотором $H(X)$, не вытекает из каких-либо общих соображений и должно рассматриваться как счастливая случайность. Полное описание таких автоморфизмов отсутствует.

Пусть сначала $(X, d)$ - пространство дискретного типа. Наложим на него следующие ограничения:

1) множество значений метрики $d$ состоит из чисел $0, \alpha^{k}, k=0,1, \ldots, 0<\alpha<1$;

2) мощность множества шаров радиуса $\alpha^{k}$ не зависит от $k$. 
Нетрудно описать все такие пространства $(X, d)$. Для этого зафиксируем множество $A$, равномощное множеству шаров пространства $(X, d)$, и обозначим через $A^{\infty}$ множество всех последовательностей $x=\left(x_{0}, x_{1}, \ldots\right), x_{i} \in A$. Введем в $A^{\infty}$ метрику $\delta$ по формулам $\delta(x, x)=0, \delta(x, y)=\alpha^{m}$, где $x=\left(x_{0}, x_{1}, \ldots\right), y=\left(y_{0}, y_{1}, \ldots\right), x \neq y$, $m=\min \left\{k \mid x_{k} \neq y_{k}\right\}$. Легко проверить, что полученное пространство $\left(A^{\infty}, \delta\right)$ изометрично исходному пространству $(X, d)$. Отсюда вытекает следующее важное для дальнейшего утверждение: шар $U_{a}^{\alpha^{k}} \subset X$ и сфера $U_{b}^{\alpha^{m}} \backslash U_{b}^{\alpha^{m+1}}$ гомотетичны для любых $a, b \in X, k, m=0,1, \ldots$, т.е. существует биективная гомотетия одного множества на другое.

Итак, возьмем пространство $(X, d)$ с описанными свойствами 1$), 2)$ и построим подгруппу $G(X, d) \subset \operatorname{Aut}(X, d)$ следуюшим образом. Возьмем точки $a, b \in X, d(a, b)=1$, и биективные гомотетии $U_{a}^{\alpha} \rightarrow X \backslash U_{b}^{\alpha}, X \backslash U_{a}^{\alpha} \rightarrow U_{b}^{\alpha}$. В результате получим биекцию $\bar{g}: X \rightarrow X$; легко видеть, что она является автоморфизмом. Обозначим через $G(X, d)$ группу автоморфизмов, порожденную автоморфизмом $\bar{g}$ и подгруппой $\operatorname{Iso}(X, d)$. Итак, искомая подгруппа $G(X, d)$ построена.

Возьмем число $\lambda>0$ и рассмотрим гильбертово пространство $H^{\left\{\beta_{k}\right\}}(X)$ (см. п. 2.2), где $\beta_{0}=1, \beta_{k}=\alpha^{2 \lambda k}\left(1-\alpha^{2 \lambda}\right)^{-1}$ при $k \geqslant 1$. Для краткости обозначим это пространство через $H_{\lambda}(X)$.

Tеорема 1. При $\lambda>0, g \in G(X, d)$ оператор $T^{\lambda}(g)$, действующий в $B(X)$ по формуле (8), продолжсается до унитарного оператора в $H_{\lambda}(X)$.

Мы сохраним обозначение $T^{\lambda}(g)$ для указанного продолжения.

ДокАЗАтЕЛЬСтво. Достаточно проверить унитарность оператора $T^{\lambda}(\bar{g})$, где $\bar{g}-$ построенный вьше автоморфизм. Напомним, что в пространстве $H_{\lambda}(X)$ имеется ортогональный базис $\left\{f^{0}, f_{c}^{k}\right\}, k \geqslant 1$, где $f^{0}(x)=1, x \in X, f_{c}^{k}$ - индикатор шара $U_{c}^{\alpha^{k}}=\left\{x \mid d(x, c) \leqslant \alpha^{k}\right\}$. Простые вычисления показывают, что образы этих функций под действием оператора $T^{\lambda}(\bar{g})$ выражаются формулами

$$
\begin{aligned}
T^{\lambda}(\bar{g}) f^{0} & =\left(\alpha^{-\lambda}-\alpha^{\lambda}\right) f_{a}^{1}+\alpha^{\lambda} f^{0}, \quad T^{\lambda}(\bar{g}) f_{b}^{1}=\alpha^{\lambda}\left(f^{0}-f_{a}^{1}\right), \\
& T^{\lambda}(\bar{g}) f_{c}^{k}=\alpha^{-\lambda} f_{c \bar{g}^{-1}}^{k+1}, \quad k \geqslant 1, \quad d(c, b)=1, \\
& T^{\lambda}(\bar{g}) f_{c}^{k}=\alpha^{\lambda} f_{c \bar{g}^{-1}}^{k-1}, \quad k \geqslant 2, \quad d(c, b) \leqslant \alpha .
\end{aligned}
$$

Несложные вычисления скалярных произведений показывают, что оператор $T^{\lambda}(\bar{g})$ унитарен. Теорема доказана.

ЗАмЕчАнИЕ. Из доказательства ясно, что утверждение теоремы 1 остается справедливым также при $\alpha^{\lambda} \in(-1,0)$. Если $\alpha^{\lambda} \in(-\infty,-1) \cup(1, \infty)$, то операторы $T^{\lambda}(g)$, $g \in G(X, d)$, унитарны относительно индефинитного скалярного произведения.

Прежде, чем закончить рассмотрение пространств дискретного типа, вернемся к конструкции автоморфизма $\bar{g}$. В этой конструкции были использованы произвольные гомотетии $U_{a}^{\alpha} \rightarrow X \backslash U_{b}^{\alpha}, X \backslash U_{a}^{\alpha} \rightarrow U_{b}^{\alpha}$. Для дальнейших целей выберем эти гомотетии специальным образом. Для этого рассмотрим множество $D=\left\{\alpha^{k}, k \in \mathbb{Z}\right\}$ и положим $X_{r}=\{x \mid d(x, b)=r\}$ при $r \in D, r<1 ; X_{r}=\left\{x \mid d(x, a)=r^{-1}\right\}$ при $r \in D$, $r>1 ; X_{1}=\{x \mid d(x, a)=d(x, b)=1\}$. Полученные множества $X_{r}, r \in D$, образуют разбиение пространства $X$ на попарно не пересекающиеся подмножества. Возьмем произвольные биективные гомотетии $X_{r} \rightarrow X_{\alpha r}, r \in D$. В результате получим 
биекцию $\bar{g}: X \rightarrow X$. Ясно, что она является частным случаем построенных вьше автоморфизмов $\bar{g}$. Добавим, что для любого целого $m$ возникают биективные гомотетии $\bar{g}^{m}: X_{r} \rightarrow X_{\gamma r}, \gamma=\alpha^{m}, r \in D$. На этом заканчивается рассмотрение пространства $(X, d)$ дискретного типа.

Обратимся к пространству $(X, d)$ непрерывного типа, $\operatorname{diam} X=1$. Рассмотрим ту же задачу: построить подгруппу $G(X, d) \subset \operatorname{Aut}(X, d)$, содержащую подгруппу Iso $(X, d)$, но не совпадающую с ней, такую, что при $\lambda>0, g \in G(X, d)$ оператор $T^{\lambda}(g)$ унитарен в одном из гильбертовых пространств $H_{\theta}^{\beta}(X)$, зависящем от $\lambda$; напомним, что пространства $H_{\theta}^{\beta}(X)$ были описаны в п. 2.2. Обозначим через $S_{a}^{r}$ сферу $\{x \mid d(x, a)=r\}$.

Наложим на $(X, d)$ следующие ограничения:

1) множество $E$, состоящее из положительных значений метрики $d$, есть полугруппа относительно умножения чисел, $1 \in E$;

2) пространство $X$ и любая сфера $S_{a}^{r}, r \in E$, гомотетичны.

Заметим, впрочем, что условие 1) вытекает из 2).

Приведем пример такого пространства. Пусть $A$ - бесконечное множество с отмеченным элементом $\bar{a}, A \frac{\infty}{a}-$ множество всех отображений $x:[0, \infty) \rightarrow A$ таких, что $x(t)=\bar{a}$ для всех $t \geqslant 0$, за возможным исключением дискретного (возможно, пустого) множества значений. Зафиксируем число $\beta \in(0,1)$ и введем в $A \frac{\infty}{a}$ метрику $\delta$ по формулам $\delta(x, x)=0, \delta(x, y)=\beta^{s}$, где $x, y \in A_{\bar{a}}^{\infty}, x \neq y, s=\inf \{t \mid x(t) \neq y(t)\}$. Полученное пространство $\left(A_{\bar{a}}^{\infty}, \delta\right)$ удовлетворяет условиям 1$\left.), 2\right)$. Здесь $E=(0,1]$.

Вернемся к произвольному пространству $(X, d)$ со свойствами 1$), 2)$ и построим обещанную подгруппу $G(X, d) \subset \operatorname{Aut}(X, d)$ и гильбертово пространство $H_{\theta}^{\beta}(X)$. Сначала построим автоморфизм $\bar{g}$ по аналогии со случаем пространств дискретного типа. Положим $D=E \cup E^{-1}$, где $E^{-1}=\left\{t \mid t^{-1} \in E\right\}$. Возьмем точки $a, b \in X, d(a, b)=1$, и введем множества $X_{r}, r \in D$, полагая $X_{r}=S_{b}^{r}$ при $r<1, X_{r}=S_{b}^{1 / r}$ при $r>1$, $X_{1}=S_{a}^{1} \cap S_{b}^{1}$. Эти множества образуют разбиение пространства $X$ на непересекающиеся части. Из указанных выше условий 1$), 2$ ) легко вьвести, что множества $X_{r}, r \in D$, попарно гомотетичны. Зафиксируем число $\gamma \in E$ и возьмем произвольные гомотетии $X_{r} \rightarrow X_{\gamma r}$. В результате получим биекцию $\bar{g}: X \rightarrow X$. Покажем, что $\bar{g} \in \operatorname{Aut}(X, d)$. Для этого возьмем кусочно-гладкую функцию $\varphi(t), 0 \leqslant t \leqslant 1$, точку $c \in X$ и рассмотрим функцию $\varphi(d(x, c)), x \in X$. Покажем, что функция $\varphi(d(x \bar{g}, c)), x \in X$, принадлежит алгебре $B(X)$. Это требует утомительного перебора ряда случаев, мы рассмотрим лишь случай $d(c, b)=r<\gamma<1$. Пусть $c^{\prime}=c \bar{g}^{-1}$. Из построения преобразования $\bar{g}$ легко вывести, что величина $d(x \bar{g}, c)$ принимает значения $1, \gamma(d(x, a))^{-1}, \gamma d(x, b), \gamma d\left(x, c^{\prime}\right)$ для точек $x$, удовлетворяющих соответственно условиям $d(x, a)<\gamma, \gamma \leqslant d(x, a) \leqslant 1$, $r \gamma^{-1} \leqslant d(x, b) \leqslant 1, d\left(x, c^{\prime}\right)=r \gamma^{-1}$. Точка $\left(d(x, a), d(x, b), d\left(x, c^{\prime}\right)\right), x \in X$, пробегает плотное подмножество множества $F \subset \mathbb{R}^{3}$, состоящего из точек $\left(t_{1}, t_{2}, t_{3}\right)$, которые удовлетворяют одному из условий:

1) $t_{2}=t_{3}=1,0 \leqslant t_{1} \leqslant 1$;

2) $t_{1}=1, r / \gamma \leqslant t_{2}=t_{3} \leqslant 1$

3) $t_{1}=1,0 \leqslant t_{3} \leqslant r / \gamma=t_{2}$;

4) $t_{1}=1,0 \leqslant t_{2} \leqslant r / \gamma=t_{3}$.

Отсюда следует, что функция $\varphi(d(x \bar{g}, c)), x \in X$, представима в виде $\Phi(d(x, a), d(x, b)$, $\left.d\left(x, c^{\prime}\right)\right)$, где $\Phi$ - непрерьвная функция на $F$. Тем самым, функция $\varphi(d(x \bar{g}, c)), x \in X$, лежит в $B(X)$. Мы доказали, что преобразование $\bar{g}$ осуществляет автоморфизм ал- 
гебры $B(X)$. Наконец, якобиан $\dot{\bar{g}}$ выражается формулами $\dot{\bar{g}}(x)=\gamma$ при $d(x, b)<1$ и при $d(x, a)=d(x, b)=1, \dot{\bar{g}}(x)=\gamma^{-1}$ при $d(x, a) \leqslant \gamma, \dot{\bar{g}}(x)=\gamma(d(x, a))^{-2}$ при $\gamma \leqslant d(x, a) \leqslant 1$. Итак, $\bar{g} \in \operatorname{Aut}(X, d)$. Через $G(X, d)$ обозначим группу автоморфизмов, порожденную автоморфизмом $\bar{g}$ и подгруппой Iso $(X, d)$. Искомая подгруппа $G(X, d)$ построена.

Зафиксируем число $\lambda>0$ и построим гильбертово пространство $H_{\theta}^{\beta}(X)$, в котором операторы $T^{\lambda}(g), g \in G(X, d)$, окажутся унитарными. Для этого рассмотрим пространства $H^{\left\{\beta_{k}\right\}}(X), \beta_{0}=1, \beta_{k}=\alpha^{2 \lambda k}\left(1-\alpha^{2 \lambda}\right)^{-1}$ при $k \geqslant 1$, построенные вьше для пространства $(X, d)$ дискретного типа. Совершим "неформальный" переход к пределу при $\alpha \rightarrow 1-0$, следуя образцу, описанному в п. 2.2. Другими словами, введем меру, сосредоточенную на множестве $\left\{\alpha^{k}, k \geqslant 0\right\}$ и предписьвающую вес $\beta_{k}\left(\alpha^{k-1}-\alpha^{k}\right)^{2}$ точке $\alpha^{k}$. Несложное вычисление, которое мы опустим, показывает, что при $\alpha \rightarrow 1-0$ эта мера слабо сходится к мере

$$
\frac{1}{2 \lambda} t^{2 \lambda+1} d t, \quad 0 \leqslant t \leqslant 1
$$

Заметим, что величина $\beta_{0}$ не зависит от $\alpha$. Теперь резонно рассмотреть гильбертово пространство $H_{\theta}^{\beta}(X)$, где $\beta=1, \theta(t)=t^{2 \lambda+1} /(2 \lambda), 0 \leqslant t \leqslant 1$. Для краткости обозначим его через $H_{\lambda}(X)$, а соответствующее скалярное произведение - через $(\cdot, \cdot)_{\lambda}$. Напомним, что для функций $f(x)=\varphi(d(x, a)), h(x)=\psi(d(x, b)), x \in X$, это скалярное произведение выражается равенством

$$
(f, h)=\varphi(1) \overline{\psi(1)}+\frac{1}{2 \lambda} \int_{r}^{1} \varphi^{\prime}(t) \overline{\psi^{\prime}(t)} t^{2 \lambda+1} d t, \quad r=d(a, b) .
$$

Tеорема 2. При $\lambda>0$ операторы $T^{\lambda}(g), g \in G(X, d)$, действующие в $B(X)$ nо формуле (8), продолжаются до унитарных операторов в гильбертовом пространстве $H_{\lambda}(X)$.

Эти продолжения обозначим также через $T^{\lambda}(g)$.

Мы вправе опустить доказательство этой теоремы: во-первых, приведенные вьше "неформальные" переходы к пределу от дискретного типа к непрерывному нетрудно превратить в строгое рассуждение, приводящее к доказательству; во-вторых, унитарность оператора $T^{\lambda}(\bar{g})$ (этого достаточно для доказательства теоремы) можно доказать явным вычислением скалярных произведений функций $T^{\lambda}(\bar{g}) f, f(x)=\varphi(d(x, c))$.

Закончим этот параграф следующей задачей: описать все автоморфизмы приведенного выше пространства последовательностей $\left(A^{\infty}, \delta\right)$, для которого оператор $T^{\lambda}(g)$ унитарен при некотором $\lambda \in \mathbb{C}$ в одном из гильбертовых пространств $H^{\left\{\beta_{k}\right\}}(X)$.

\section{§4. Вполне-бесконечные пространства, связанные}

\section{с полем формальных рядов. Представления группы $\mathrm{GL}_{2}(\mathbb{F})$}

Мы рассмотрим пример вполне-бесконечного пространства. Предварительно введем обозначения. Пусть $\mathbb{K}$ - бесконечное поле, $\mathbb{F}$ - множество формальных рядов

$$
\xi=\sum f(t) z^{t}
$$

где $t \in \mathbb{R}, f: \mathbb{R} \rightarrow \mathbb{K}$ - произвольная функция такая, что множество $\{t \mid f(t) \neq 0\}$ ограничено снизу и дискретно (возможно, пустое). Обычное умножение рядов превращает $\mathbb{F}$ 
в поле. Введем в $\mathbb{F}$ норму $\|\xi\|=\alpha^{s}, s=\inf \{t \mid f(t) \neq 0\}, 0<\alpha<1$. Через $\mathbb{F}^{0}$ обозначим подполе рядов

$$
\sum_{-\infty}^{\infty} a_{n} z^{n}, \quad a_{n} \in \mathbb{K}
$$

$a_{n}=0$ при отрицательных значениях числа $n$ для достаточно больших $|n|$. Наконец, через $\mathrm{GL}_{2}(\mathbb{F}), \mathrm{GL}_{2}\left(\mathbb{F}^{0}\right)$ обозначим группы невырожденных матриц второго порядка с элементами из $\mathbb{F}, \mathbb{F}^{0}$.

Определим вполне-бесконечное пространство. Пусть $\mathbb{P}^{1}(\mathbb{F})$ - проективная прямая над $\mathbb{F}$; элемент пространства $\mathbb{P}^{1}(\mathbb{F})$ - множество $x=\left\{\left(\lambda x_{1}, \lambda x_{2}\right), \lambda \neq 0\right\}$, где $\left(x_{1}, x_{2}\right) \in$ $\mathbb{F}^{2} \backslash\{(0,0)\}$. Этот элемент обозначим через $x_{1}: x_{2}$. Введем в $\mathbb{P}^{1}(\mathbb{F})$ метрику $d$ по формуле $d(x, y)=\left\|x_{1} y_{2}-x_{2} y_{1}\right\|$, где $x=x_{1}: x_{2}, y=y_{1}: y_{2}, \max _{i}\left\|x_{i}\right\|=1, \max _{i}\left\|y_{i}\right\|=1$. Получим пространство непрерьвного типа $\left(\mathbb{P}^{1}(\mathbb{F}), d\right)$. Аналогично определяется пространство $\left(\mathbb{P}^{1}\left(\mathbb{F}^{0}\right), d\right)$.

Группа $\mathrm{GL}_{2}(\mathbb{F})$ действует на $\mathbb{F}^{2}$ по формуле

$$
\left(\xi_{1}, \xi_{2}\right) g=\left(\xi_{1} a+\xi_{2} c, \xi_{1} b+\xi_{2} d\right), \quad g=\left(\begin{array}{cc}
a & b \\
c & d
\end{array}\right) \in \mathrm{GL}_{2}(\mathbb{F})
$$

Это приводит к действию $x \mapsto x g, x \in \mathbb{P}^{1}(\mathbb{F}), g \in \mathrm{GL}_{2}(\mathbb{F})$.

Лемма 4. Указанное действие группь $\mathrm{GL}_{2}(\mathbb{F})$ на $\mathbb{P}^{1}(\mathbb{F})$ задает гомоморфизм $\mathrm{GL}_{2}(\mathbb{F}) \rightarrow G\left(\mathbb{P}^{1}(\mathbb{F}), d\right)$. Аналогичное утвержсдение справедливо и для $\mathrm{GL}_{2}\left(\mathbb{F}^{0}\right)$.

ДокАЗАтЕльство. Группа $\mathrm{GL}_{2}(\mathbb{F})$ порождается подгруппой $\mathrm{GL}_{2}(\overline{\mathbb{F}})$, где $\overline{\mathbb{F}}=\{\xi \mid$

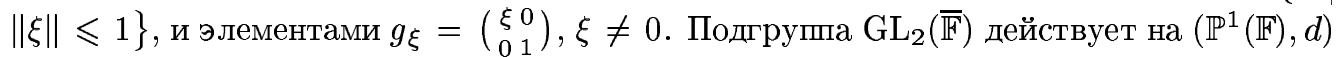
изометрически. Легко проверяется, что матрица $g_{\xi}$ действует на $\left(\mathbb{P}^{1}(\mathbb{F}), d\right)$ точно так, как преобразование $\bar{g}$, построенное в п. 3.2. Лемма доказана.

Добавим лишь, что в качестве точек $a, b$, использованных при построении преобразования $\bar{g}$, следует взять $0: 1,1: 0$.

Используя гомоморфизмы, указанные в лемме 4, и теоремы 1,2 , получаем унитарные представления групп $\mathrm{GL}_{2}(\mathbb{F}), \mathrm{GL}_{2}\left(\mathbb{F}^{0}\right)$; coхраним для них обозначение $T^{\lambda}$. Приведем явные формулы, задаюшие эти представления. Представления групшы $\mathrm{GL}_{2}\left(\mathbb{F}^{0}\right)$ определяются числом $\lambda>0$ и действуют в пространстве $H^{\left\{\beta_{k}\right\}}\left(\mathbb{P}^{1}(\mathbb{F})\right)$, $\beta_{0}=1, \beta_{k}=\alpha^{2 \lambda k}\left(1-\alpha^{2 \lambda}\right)^{k}, k \geqslant 1$, по формуле

$$
\left(T^{\lambda}(g) f\right)(x)=\|\operatorname{det} g\|^{\lambda} \cdot\|\bar{x} g\|^{-2 \lambda} f(x g), \quad x=x_{1}: x_{2}, \quad \bar{x}=\left(x_{1}, x_{2}\right), \quad \max _{i}\left\|x_{i}\right\|=1 .
$$

(Здесь $\|y\|=\max _{i}\left\|y_{i}\right\|, y=\left(y_{1}, y_{2}\right) \in \mathbb{F}^{2}$.)

Наконец, представление $T^{\lambda}, \lambda>0$, групшы $\mathrm{GL}_{2}(\mathbb{F})$ действует в пространстве $H_{\theta}^{\beta}\left(\mathbb{P}^{1}(\mathbb{F})\right), \beta=1, \theta(t)=t^{2 \lambda+1} /(2 \lambda)$, по той же формуле $(9)$.

Возможно, рассмотренные в этой работе пространства $H^{\left\{\beta_{k}\right\}}, H_{\theta}^{\beta}$ окажутся полезными при построении представлений других “больших” групп. 


\section{СПИСОК ЦИТИРОВАННОЙ ЛИТЕРАТУРЫ}

[1] Вестфрид И.А., Тиман А.Ф. Об одном свойстве универсальности гильбертовых пространств // Докл. АН СССР. 1979. Т. 246. № 3. С. 528-530.

[2] Вестфрид И.А. Об универсальных ультраметрических пространствах // Укр. матем. ж. 1994. Т. 46. №12. С. 1700-1706.

[3] Исмагилов Р. С. О неприводимых представлениях дискретной группы $\mathrm{SL}_{2}(\mathbb{P})$, унитарных в индефинитной метрике // Изв. АН СССР. Сер. матем. 1966. Т. 30. № 4. С. 923-950.

[4] Исмагилов Р. С. Элементарные сферические функции на группе $\mathrm{SL}_{2}(\mathbb{P})$ над полем, не являющимся локально компактным // Изв. АН СССР. Сер. матем. 1967. Т. 31. № 2. С. 361-390.

[5] Исмагилов Р. С. О линейных представлениях групп матриц с элементами из нормированного поля // Изв. АН СССР. Сер. матем. 1969. Т. 33. №6. С. 1296-1323.

[6] Исмагилов Р. С. О представлениях $\mathrm{SL}_{2}(\mathbb{P})$, где $\mathbb{P}$ не локально компактно // Функцион. анализ и его прилож. 1973. Т. 7. № 4. С. 85-86.

[7] Тиман А.Ф. Об аппроксимативных характеристиках метрических пространств и приближении функций // Тр. МИАН. 1975. Т. 134. С. 314-326.

Московский государственный технический университет

Поступило

им. Н.Э. Баумана

06.03 .96 